Bull. Chem. Soc. Ethiop. 2013, 27(3), 387-393.

Printed in Ethiopia

DOI: http://dx.doi.org/10.4314/bcse.v27i3.7

ISSN 1011-3924

(c) 2013 Chemical Society of Ethiopia

\title{
SYNTHESIS AND CHARACTERIZATION OF CdTe QUANTUM DOTS BY ONE-STEP METHOD
}

\author{
Yilin Wang ${ }^{* 1,2}$, Rongfang Wang ${ }^{2}$, Shengyan $\mathrm{Liu}^{2}$, Kun Yang ${ }^{2}$, Liya Zhou ${ }^{2}$ and Huaimei $\mathrm{Li}^{2}$ \\ ${ }^{1}$ Ministry-province jointly-constructed cultivation base for state key laboratory of Processing for \\ non-ferrous metal and featured materials, Guangxi Zhuang Autonomous Region, Guangxi \\ University, Nanning 530004, China \\ ${ }^{2}$ School of Chemistry and Chemical Engineering, Guangxi University, Nanning 530004, China
}

(Received September 18, 2012; revised May 7, 2013)

\begin{abstract}
L-Cysteine (Cys)-capped CdTe quantum dots (QDs) were prepared when sodium tellurite worked as a tellurium source and sodium borohydride acted as a reductant. The influences of various experimental variables, including $\mathrm{pH}$ values, $\mathrm{Cd} / \mathrm{Te}$ and $\mathrm{Cd} / \mathrm{Cys}$ molar ratios, on the photoluminescence (PL) quantum yield (QY) of the obtained CdTe QDs have been systematically investigated. Experimental results indicated that green to red emitting CdTe QDs with maximum quantum yield of $19.4 \%$ can be prepared at $\mathrm{pH} 11.5$ and $\left.\left.n_{(\mathrm{Cd}}{ }^{2+}\right): n_{(\mathrm{Te}}{ }^{2-}\right): n_{(\mathrm{Cys})}=1: 0.07: 2.0$. X-Ray powder diffraction (XRD) and transmission electron microscopy (TEM) were used to characterize the crystal structure and shape of CdTe QDs. The results showed that the prepared CdTe QDs were of cubic zinc blend crystal structure in a sphere-like shape.
\end{abstract}

KEY WORDS: Semicondutor nanocrystal, Chemical synthesis, Optical properties, Luminescence

\section{INTRODUCTION}

In recent years, with the development of the preparing technology, semiconductor quantum dots (QDs) have been widely used in the research of biomedicine [1,2] and optoelectronic devices $[3,4]$ because of their unique optical properties. So far, two successful routes, including organic synthesis [5, 6] and aqueous synthesis [7, 8], have been developed for the preparation of CdTe QDs. Compared with organic synthesis, aqueous synthesis is more reproducible, cheaper, less toxic, and the as-prepared QDs are more water-soluble and bio-compatible. Several improvements of the conventional aqueous synthetic method for thiol-capped CdTe QDs have been reported recently by different groups, including the hydrothermal synthesis, ultrasonic or microwave irradiation [9]. However, in most of these aqueous synthesis, Te powder [10, 11] or $\mathrm{Al}_{2} \mathrm{Te}_{3}$ lump $[12,13]$ is often used as tellurium sources, which needs a pretreatment to synthesize the instable tellurium precursor $\left(\mathrm{NaHTe}\right.$ or $\mathrm{H}_{2} \mathrm{Te}$ ) and the process of preparing CdTe QDs requires nitrogen as the protective gas at the initial stage. In our previous report $[14,15]$, we have provided an alternative approach to prepare $\mathrm{CdTe}$ QDs using $\mathrm{TeO}_{2}$ as the Te source. In this work, we synthesized Cys-CdTe QDs in air conditions, using $\mathrm{Na}_{2} \mathrm{TeO}_{3}$ as Te source and $\mathrm{NaBH}_{4}$ as reductant. The influences of the precursor $\mathrm{Cd} / \mathrm{Te}$ molar ratios, Cd/Cys molar ratios and the $\mathrm{pH}$ of original solution on quantum yield of the obtained CdTe QDs have been systematically investigated. The crystal structure and shape of CdTe QDs were characterized by XRD and TEM.

*Corresponding author. E-mail: theanalyst@163.com 


\section{EXPERIMENTAL}

\section{Reagents and materials}

L-Cysteine hydrochloride monohydrate (99\%), $\mathrm{Na}_{2} \mathrm{TeO}_{3}(97 \%), \mathrm{CdCl}_{2} \cdot 2.5 \mathrm{H}_{2} \mathrm{O}$ (99\%) and $\mathrm{NaBH}_{4}(96 \%$ ), (all from Sinopharm Chemical Reagent Co., Ltd, Shanghai, China) were used for the preparation of CdTe. Rhodamine $6 \mathrm{G}$ with a photoluminescence quantum yield (PLQY) of 95\% in water was obtained from Aladdin Chemistry Co., Ltd (China) to determine quantum yields of QDs. All chemicals were used as received without further purification. Double deionized water was used throughout the experiments.

\section{Preparation of CdTe QDs}

The water soluble Cys-CdTe QDs were one-step synthesized by using $\mathrm{Na}_{2} \mathrm{TeO}_{3}, \mathrm{NaBH}_{4}$ and $\mathrm{CdCl}_{2} \cdot 2.5 \mathrm{H}_{2} \mathrm{O}$ as precursors. Typically, $0.7025 \mathrm{~g}$ L-cysteine hydrochloride monohydrate was added to $100 \mathrm{~mL}$ of $0.02 \mathrm{M} \mathrm{CdCl}_{2}$ solution under stirring. Then, the solution was adjusted to $\mathrm{pH}$ 11.5 by dropwise addition of $1 \mathrm{M} \mathrm{NaOH}$ solution. The $\mathrm{NaBH}_{4}(0.1 \mathrm{~g})$ and $\mathrm{Na}_{2} \mathrm{TeO}_{3}(0.0323 \mathrm{~g})$ were added to the solution under stirring. Afterward, the resulting mixture solution was heated to $100{ }^{\circ} \mathrm{C}$ and refluxed at $10,30,60,180,300$ and $420 \mathrm{~min}$ for controlling the size of CdTe QDs.

\section{Characterization}

After the samples were diluted, the absorption and photoluminescence (PL) spectra were measured using a UV-2102PC spectrophotometer and a RF-5301 fluorescence spectrophotometer, respectively. The as-prepared QDs were precipitated for 10 min by adding three-fold volume isopropyl alcohol, and the sediment was collected after centrifugation at 4000 rpm for $5 \mathrm{~min}$. After drying, the obtained powder was used for XRD analysis. The sample powder was placed onto sample holder plates, and the XRD spectra was carried out by using a Rigaku/Dmax-2500 X-ray diffractometer with $\mathrm{Cu} K \alpha$ radiation $(\lambda=1.5406 \AA)$. The QDs solution was spread on ultra-thin carbon-coated film on 200 mesh copper grids and left to dry in air. Sample was then visualized using a FEI-TF30 transmission electron microscopy.

\section{Measurement of quantum yields}

The PLQY was obtained by comparison with rhodamine 6G $(\mathrm{QY}=95 \%)$ and using data derived from the luminescence and the absorption spectra [16]. Briefly, rhodamine $6 \mathrm{G}$ was chosen as a reference standard $(\mathrm{QY}=95 \%)$, the absorbance for the standard and the CdTe QDs samples at the excitation wavelength and the fluorescence spectra of the same solutions were measured, respectively. The expression to calculate quantum yield is provided as following:

$$
Y_{\mathrm{u}}=Y_{\mathrm{s}} \times \frac{F_{\mathrm{u}} \times A_{\mathrm{s}}}{F_{\mathrm{s}} \times A_{\mathrm{u}}}
$$

In Eq.(1), $Y$ is quantum yield, $F$ is the integrated emission peak area, and $A$ is the absorbance at excitation wavelength. The subscripts $s$ and $u$ denote standard (such as rhodamine 6G) and test samples, respectively. The absorbance at the excitation wavelength $(365 \mathrm{~nm})$ of rhodamine $6 \mathrm{G}$ and the QDs sample was set to a similar value (below 0.1). 


\section{RESULTS AND DISCUSSION}

\section{$U V-$ Vis absorption and PL spectra}

Figure 1 shows the absorption (solid line) and PL (dashed line) spectra of the typical Cys-CdTe QDs. The excitation absorption peaks of the QDs are quite obvious, with reflux from 10 to 420 min, the maximum absorption peaks appeared at 490, 508, 521, 584, 561 and $579 \mathrm{~nm}$, respectively. Compared with CdTe bulk material $(827 \mathrm{~nm})$ [17], the excitation absorption peaks of CdTe QDs have significant blue shift, which showing obvious quantum confinement effect. The maximum absorption peak of QDs took place an obvious red-shift from $490 \mathrm{~nm}$ to $579 \mathrm{~nm}$ with increasing size of the QDs as a consequence of the quantum confinement. According to the empirical formula [18]:

$D=\left(9.8127 \times 10^{-7}\right) \lambda^{3}-\left(1.7147 \times 10^{-3}\right) \lambda^{2}+(1.0064) \lambda-194.84$

where $\lambda$ is the first absorption maximum, the diameter of samples was estimated to 2.0, 2.5, 2.9, 3.2, 3.3 and $3.5 \mathrm{~nm}$, respectively. Similar to absorption spectra, PL emission spectra also take place a red shift with the increasing refluxing time. In addition, the PL emission spectra are located close to the absorption thresholds, indicating that the PL of CdTe QDs is from the excitation luminescence of elections between valence band and conduction band transitions [13]. The full width of half maximum of PL spectra is between $30 \mathrm{~nm}$ and $55 \mathrm{~nm}$, meaning that the particle sizes are evenly distributed.

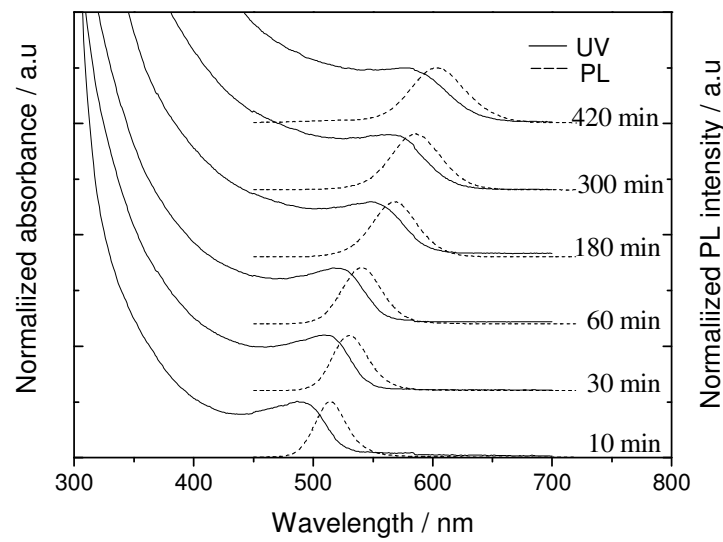

Figure 1. Temporal evolution of UV-Vis absorption (solid line) and PL spectra (dashed line) of CdTe QDs.

$X R D$ and TEM

The XRD pattern of the as-prepared CdTe QDs that was refluxed for $180 \mathrm{~min}$ at $100{ }^{\circ} \mathrm{C}$ is shown in Figure 2. The diffraction peaks at $2 \theta=24.535^{\circ}, 40.589^{\circ}$, and $48.037^{\circ}$ can be readily assigned to the (1 111$),\left(\begin{array}{lll}2 & 2 & 0\end{array}\right)$, and ( $\left.\begin{array}{lll}3 & 1 & 1\end{array}\right)$ planes, respectively. The position of the XRD peaks matches well those of the bulk CdTe cubic structure (JCPDS No.65-1046). These peaks are comparatively wider than that of the bulk materials due to the finite crystalline size.

The TEM image of corresponding sample was shown in Figure 3. The CdTe QDs appear as spherical particles with the average size of about $3.0 \mathrm{~nm}$, which was in an agreement with that 
calculated from the UV-Vis absorption spectra, and the sample were mono-disperse and homogeneous.

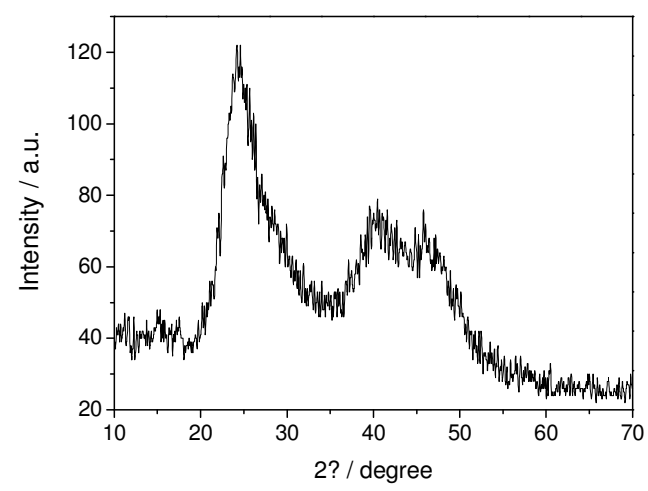

Figure 2. The XRD pattern of CdTe QDs.

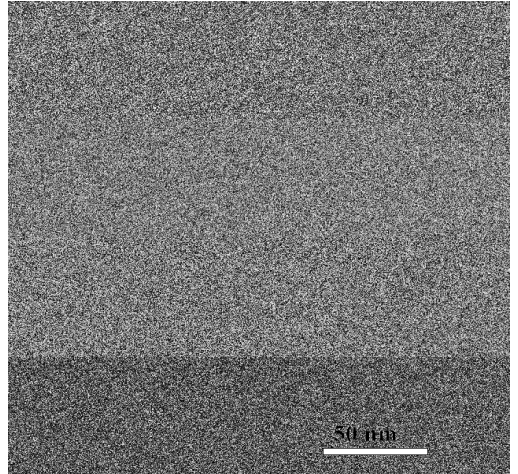

Figure 3. TEM image of CdTe QDs.

\section{Influence of $\mathrm{pH}$ value, $\mathrm{Cd} / \mathrm{Te}$ and $\mathrm{Cd} / \mathrm{Cys}$ molar ratios on optical properties}

It has been demonstrated that cadmium and thiol can form different polynuclear compounds in water and that the formation of cadmium thiol compounds strongly depends on $\mathrm{pH}$ of the solution [19]. When the $\mathrm{pH}$ is less than 4.0, there is little cadmium thiol compound existed. Consequently, the $\mathrm{pH}$ of solution should be controlled in a certain range when CdTe QDs are synthesized in aqueous solution whose stabilizer is thiol, to ensure enough cadmium thiol compound existing. In the experiment, the initial $\mathrm{pH}$ values was adjusted to $10.5,11.0$ and 11.5 under the molar ratio of $\mathrm{Cd}^{2+} / \mathrm{Te}^{2-} / \mathrm{Cys}$ was $1: 0.05: 2$, respectively, we investigated the influence of various initial $\mathrm{pH}$ values on the PL QYs of CdTe QDs. It can be seen in Figure 4, being refluxed for $60 \mathrm{~min}$, the highest quantum yields of QDs was obtained in three different $\mathrm{pH}$ values up to $13.2 \%, 14.3 \%$ and $16.2 \%$, respectively. In addition, the quantum yields of the corresponding reflux time were improved with the $\mathrm{pH}$ of original solution increase. It could be easier to form $\mathrm{Cd}^{2+}$-Cys coordination bond higher while reducing surface defects or a thin layer of $\mathrm{CdS}$ shell was formed to passivate the quantum dots surface, and improve the luminous efficiency with higher $\mathrm{pH}$. Experimental results show that the appropriate $\mathrm{pH}$ of synthesizing Cys-CdTe QDs is 11.5.

The $\mathrm{Cd} / \mathrm{Te}$ molar ratios also played an important role in improvement of quantum yield of CdTe QDs. In previous reports [20-22], different $\mathrm{Cd} / \mathrm{Te}$ molar ratios (changing from 1:0.2 to 1:0.5) were adopted in the aqueous synthesis of CdTe QDs using Te powder or $\mathrm{Al}_{2} \mathrm{Te}_{3}$ lump as tellurium source. In the present study, the $\mathrm{Cd} / \mathrm{Te}$ molar ratio was varied from 1:0.05 to 1:0.2 with fixed $\mathrm{Cd} / \mathrm{Cys}$ molar ratio at $1: 2$ and $\mathrm{pH}$ value at 11.5 , we investigated the influence of various $\mathrm{Cd} / \mathrm{Te}$ molar ratios on the PLQY of CdTe QDs, and the obtained experimental results are shown in Figure 5. It can be seen that the CdTe QDs prepared at Cd/Te molar ratio 1:0.07 possess a high PLQY value, and the maximum quantum yield up to $19.4 \%$. While the PLQY value of the resulting CdTe QDs were relatively low at the $\mathrm{Cd} / \mathrm{Te}$ molar ratio 1:0.2. The literature report [23] revealed that highly luminescent CdTe QDs possess much fewer Te atoms on the surface than that of CdTe with low luminescence. Here, we suppose that, with the fixed amount of $\mathrm{Cd}$ and Cys, increasing the amount of $\mathrm{Te}$, the relative coverage rate of the particle surface with Te atoms will increase, which results in the low luminescence. Herein, a proper $\mathrm{Cd} / \mathrm{Te}$ molar ratio is important for preparation of high quality CdTe QDs, in our experiment, the optimal ratio is 1:0.07. 


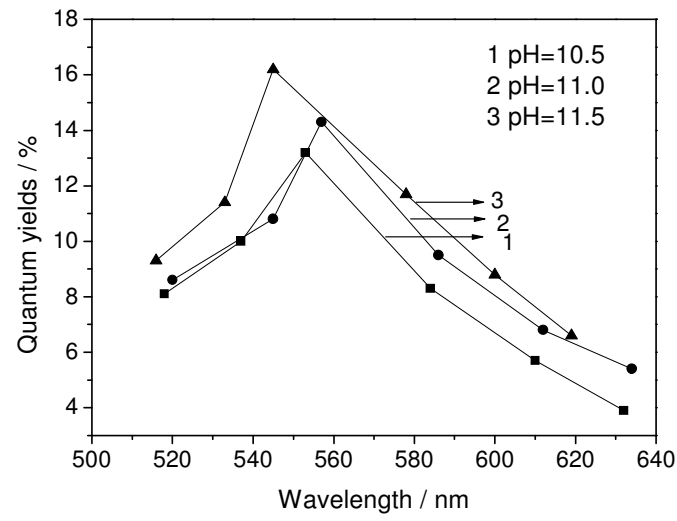

Figure 4. QY of CdTe QDs at different emission peaks as a function of $\mathrm{pH}$. From left to right, the QDs are obtained for refluxing 10, 30, 60, 180, 300 and $420 \mathrm{~min}$.

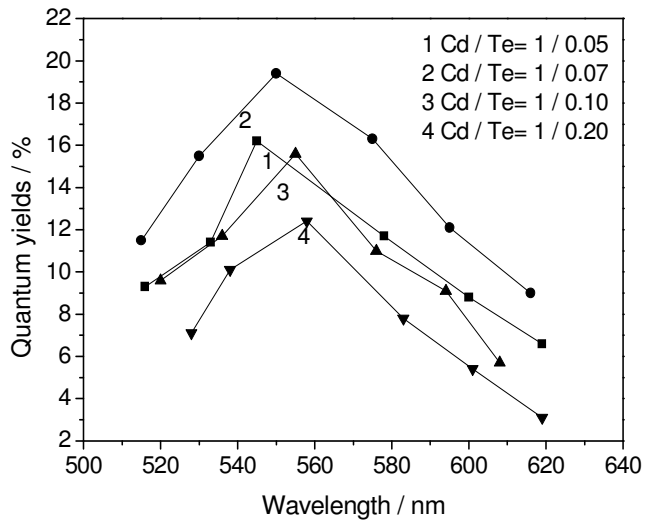

Figure 5. QY of CdTe QDs prepared at different Cd/Te molar ratios. From left to right, the QDs are obtained for refluxing 10, 30, 60, 180, 300 and $420 \mathrm{~min}$.

As previously demonstrated, the occupation of surface sites by stabilizer molecules instead of $\mathrm{Te}$ atoms has a tremendous contribution in removing the dangling bonds and preventing the oxidation of Te atoms, which is greatly favorable for the formation of high-quality QDs [24]. Therefore, the thiol plays an important role in the synthesis of thiol-capped CdTe QDs, and in most of the "traditional" aqueous approaches, the stoichiometric ratio of $\mathrm{Cd}$ to thiol was often set as $1: 2.45[13,25]$. In our experiments, the $\mathrm{Cd} / \mathrm{Cys}$ molar ratio was changed from 1:1.6 to 1:2.2 with fixed $\mathrm{Cd} / \mathrm{Te}$ molar ratio (1:0.07) and $\mathrm{pH}$ value (11.5), we investigated the influence of various $\mathrm{Cd} / \mathrm{Cys}$ molar ratios on the PLQY of CdTe QDs. As can be seen in Figure 6, when the $\mathrm{Cd} / \mathrm{Cys}=1 / 1.8$, the PLQY of the resulting CdTe QDs were relatively low due to the absence of adequate Cys molecules. The population of Cys molecules on the surface of QDs was increased, accompanied by decrease of the Cd/Cys molar ratio, thus, the PLQY was gradually enhanced and up to the maximum while the $\mathrm{Cd} / \mathrm{Cys}$ molar ratio reached the optimum value of 1:2. However, an excessive number of stabilizer molecules might distort the surface, consequently, the rough surface gave rise to new nonradiative defects. As a result, the PLQY 
was distinctly diminished when the $\mathrm{Cd} / \mathrm{Cys}=1 / 2$. Therefore, the $\mathrm{Cd} / \mathrm{Cys}$ molar ratio $1: 2.0$ is optimal for the synthesis of Cys-CdTe QDs.

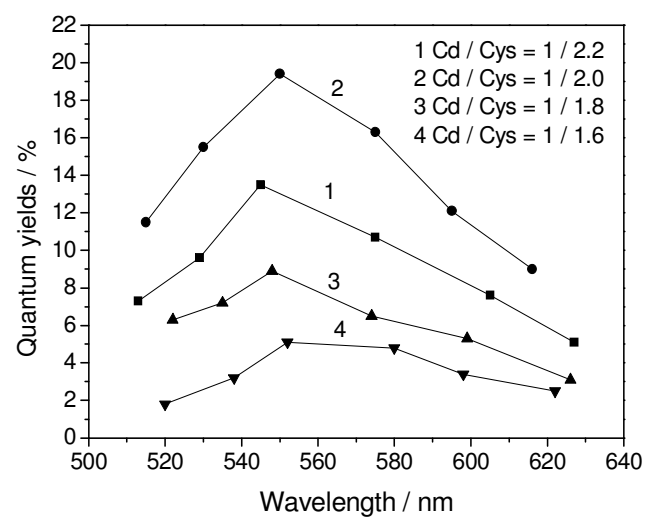

Figure 6. QY of CdTe QDs prepared at different Cd/Cys molar ratios. From left to right, the QDs are obtained for refluxing 10, 30, 60, 180, 300 and $420 \mathrm{~min}$.

Mechanism for the preparation of CdTe QDs

The formation of QDs in solution often involves in two stages: nucleation and growth. In this synthesis process, the following mechanism is proposed to elaborate the formation of CdTe QDs [9]: firstly, cadmium thiol complexes $\left(\mathrm{Cys}-\mathrm{Cd}^{2+}\right)$ were formed in the strong alkaline solution (Eq. 3), when the $\mathrm{TeO}_{3}{ }^{2-}$ was introduced to the three-neck flask, it can be reduced by $\mathrm{NaBH}_{4}$ to produce $\mathrm{Te}^{2-}$ (Eq. 4) that reacted with the Cys-Cd ${ }^{2+}$ complexes, resulting in the formation of CdTe nuclear (Eq. 5). Finally, with the CdTe nuclear growing gradually, the CdTe QDs was formed under the condition of reflux (Eq. 6).

$\mathrm{Cd}^{2+}+\mathrm{Cys} \rightarrow \mathrm{Cys}-\mathrm{Cd}^{2+}$

$4 \mathrm{TeO}_{3}{ }^{2-}+3 \mathrm{BH}_{4}^{-} \rightarrow 4 \mathrm{Te}^{2-}+3 \mathrm{BO}_{2}^{-}+6 \mathrm{H}_{2} \mathrm{O}$

Cys $-\mathrm{Cd}^{2+}+\mathrm{Te}^{2-} \rightarrow$ Cys $-\mathrm{CdTe}$

$n$ Cys $-\mathrm{CdTe} \rightarrow(\text { Cys }-\mathrm{CdTe})_{\mathrm{n}}$

\section{CONCLUSIONS}

We developed a technology to synthesize Cys-CdTe QDs by one-step method, using $\mathrm{Na}_{2} \mathrm{TeO}_{3}$ as Te source and $\mathrm{NaBH}_{4}$ as reductant. The green to red emitting CdTe QDs with maximum quantum yield of $19.4 \%$ can be prepared at $\mathrm{pH} 11.5$ and $\left.n_{(\mathrm{Cd}}{ }^{2+}: n_{(\mathrm{Te}}{ }^{2-}\right): n_{(\mathrm{Cys})}=1: 0.07: 2.0$. Compared with Te powder and $\mathrm{Al}_{2} \mathrm{Te}_{3}$ lump route, the $\mathrm{Na}_{2} \mathrm{TeO}_{3}$ route is an environmentfriendly and low-cost synthesis route, which is suited for large-scale synthesis.

\section{ACKNOWLEDGMENT}

This work was supported by ministry-province jointly-constructed cultivation base for state key laboratory of processing for non-ferrous metal and featured materials, Guangxi Zhuang Autonomous Region (GXKFJ12-18).

\section{REFERENCES}

Bull. Chem. Soc. Ethiop. 2013, 27(3) 
1. Chan, Y.H.; Ye, F.; Gallina, M.E.; Zhang, X.J.; Jin, Y.H.; Wu, C.; Chiu, D.T. J. Am. Chem. Soc. 2012, 134, 7309.

2. Shen, X. F. Micro. Nano. Lett. 2012, 7, 137.

3. Xu, X.Q.; Giménez, S.; Mora-SeróI.; Bisquert, J.; Xu, G. Mater. Chem. Phys. 2010, 124, 709.

4. Buhbut, S.; Itzhakov, S.; Oron, D.; Zaban, A. J. Phys. Chem. Lett. 2011, 2, 1917.

5. Xing, B.; Li, W.W.; Dou, H J.; Sun, K. Chem. J. Chin. Univ. 2008, 29, 230.

6. Xu, C.F.; Kong, A.G.; Ding, H.M.; Shan, Y. Mater. Lett. 2012, 82, 44.

7. Chen, X.F.; Zhou, M.; Chang, Y.P.; Ren, C.L.; Chen, H.L.; Chen, X.G. Appl. Surf. Sci. 2012, $263,491$.

8. Sheng, Z.H.; Han, H.Y.; Hu, X.F.; Chi, C. Dalton. Trans. 2010, 39, 7017.

9. Wang, Y.L.; Liu, S.Y. J. Chil. Chem. Soc. 2012, 58, 827.

10. Wang, X.D.; Yin, P.F.; Gong, H.P.; Li, P.P.; Liu, Z.Q.; He, Y.Q. Chem. J. Chin. Univ. 2012, 33, 1182.

11. Cheng, F.; Liu, Z.; Hu, Y.Z.; Wu, S.M.; Yan, C.Y. Chem. J. Chin. Univ. 2012, 33, 475.

12. Mandal, A.; Tamai, N.T. J. Phys. Chem. C 2008, 112, 8244.

13. Gaponik, N.; Talapin, D.V.; Rogach, A.L.; Hoppe, K.; Shevchenko, E.V.; Kornowski, A.; Eychmuller, A.; Weller, H. J. Phys. Chem. B 2002, 106, 7177.

14. Wang, Y. L.; Tong, Z.F.; Lu, J.P.; Li, J.H. CIESC J. 2011, $62,875$.

15. Wang, R.F.; Wang, Y.L.; Feng, Q.L.; Zhou, L.Y.; Gong, F.Z.; Lan, Y.W. Mater. Lett. 2012, 66, 261.

16. Wang, Y. L.; Lu, J.P.; Tong, Z.F.; Li, B.; Zhou, L.Y. Bull. Chem. Soc. Ethiop. 2011, 25, 393.

17. Murray, C. B.; Norris, D.J.; Bawendi, M.G. J. Am. Chem. Soc. 1993,115, 8706.

18. Yu, W.W.; Qu, L.H.; Guo, W.Z.; Peng, X.G. Chem. Mater. 2003, 15, 2854.

19. Hayes, D.; Micic, O.I.; Nenadovic, M.T.; Swayambunathan, V.; Meisel, D. J. Phys. Chem. 1989, 93, 4603.

20. Liu, Y. F.; Yu, J. S. J. Colloid. Interf. Sci. 2009, 333, 690.

21. Feng, X.J.; Shang, Q.K.; Liu, H.J.; Wang, W.L.; Wang, Z.D.; Liu, J. Y. J. Lumin. 2010, 130, 648.

22. Zhang, T.L.; Sun, X.Y.; Liu, B. Spectrochim. Acta A 2011, 79, 1566.

23. Borchert, H.; Talapin, D.V.; Gaponik, N. J. Phys. Chem. B 2003, 107, 9662.

24. He, Y.; Lu, H.; Sai, L.; Lai, W.Y.; Fan, Q.L.; Wang, L.H.; Huang, W. J. Phys. Chem. B 2006, 110, 13352.

25. Zhang, Y.H.; Zhang, H.S.; Ma, M. App.l Surf. Sci. 2009, 255, 4747. 